\title{
The first multi-wavelength campaign of AXP 4U 0142+61 from radio to hard $\mathrm{X}$-rays
}

\author{
P.R. den Hartog • L. Kuiper • W. Hermsen • N. Rea • \\ M. Durant • B. Stappers • V.M. Kaspi $\cdot$ R. Dib
}

Received: 14 July 2006 / Accepted: 3 November 2006 / Published online: 21 March 2007

(C) Springer Science+Business Media B.V. 2007

\begin{abstract}
For the first time a quasi-simultaneous multiwavelength campaign has been performed on an Anomalous X-ray Pulsar from the radio to the hard X-ray band. 4U 0142+61 was an INTEGRAL target for $1 \mathrm{Ms}$ in July 2005. During these observations it was also observed in the X-ray band with Swift and RXTE, in the optical and NIR with Gemini North and in the radio with the WSRT. In this paper we present the source-energy distribution. The spectral results obtained in the individual wave bands do not connect smoothly; apparently components of different origin contribute to the total spectrum. Remarkable is that the INTEGRAL hard X-ray spectrum (power-law index $0.79 \pm 0.10$ ) is now measured up to an energy of $\sim 230 \mathrm{keV}$ with no indication of a spectral break. Extrapolation of the INTEGRAL power-law spectrum to lower energies passes orders of magnitude underneath the NIR and optical fluxes,
\end{abstract}

P.R. den Hartog $(\varangle) \cdot$ L. Kuiper · W. Hermsen · N. Rea SRON Netherlands Institute for Space Research, Sorbonnelaan 2, 3584 CA Utrecht, The Netherlands

e-mail: hartog@sron.nl

W. Hermsen · B. Stappers

Astronomical Institute, University of Amsterdam, Kruiskade 403, 1098 SJ Amsterdam, The Netherlands

\section{Durant}

Department of Astronomy \& Astrophysics, University of Toronto, 60 Saint George Street, MP 1404D Toronto, ON M5S 3H8, Canada

\section{B. Stappers}

ASTRON, The Netherlands Foundation for Research in Astronomy, P.O. Box 2, 7990 AA, Dwingeloo, The Netherlands

V.M. Kaspi · R. Dib

Physics Department, McGill University, 3600 University Street, Montreal, PQ H3A 2T8, Canada as well as the low $\sim 30 \mu \mathrm{Jy}(2 \sigma)$ upper limit in the radio band.

Keywords Neutron stars · Magnetars · Anomalous X-ray pulsars

PACS 97.60.Jd $\cdot 97.60 . \mathrm{Gb} \cdot 95.85 . \mathrm{Pw} \cdot 95.85 . \mathrm{Nv} \cdot$ 95.85. $\mathrm{Kr} \cdot 95.85 . \mathrm{Jq} \cdot 95.85 . \mathrm{Bh}$

\section{Introduction}

Anomalous X-ray Pulsars (AXPs) are young rotating isolated neutron stars (for a review in this volume, see Kaspi 2007). Currently there are 8 AXPs known and there are a few more candidates (Woods and Thompson 2006). These objects are called anomalous, because their X-ray luminosities exceed by far the available total energy released by rotational energy loss. The energy output is believed to originate from an immense energy reservoir stored in a toroidal magnetic field within the Neutron Star. The surface magnetic fields, inferred from their periods and period derivatives, are of the order of $10^{14}-10^{15} \mathrm{G}$. Therefore, AXPs are believed to be magnetars, as originally proposed for the Soft Gammaray Repeaters (SGRs, see Duncan and Thompson 1992; Thompson and Duncan 1995, 1996). Both AXPs and SGRs are well studied objects in the X-ray band for energies below $10 \mathrm{keV}$. However, little was known about their persistent emission in the hard X-ray band (>10 keV). In 2004 INTEGRAL discovered hard X-ray emission from the position of 1E 1841-045 (Molkov et al. 2004). Kuiper et al. (2004) showed unambiguously that the hard X-rays originated from the AXP by extracting a pulsed hard X-ray signal from the source using archival RXTE data. After INTEGRAL discovered hard X-rays from two other AXP 
locations, namely from 1RXS J1708-4009 (Revnivtsev et al. 2004) and 4U 0142+61 (den Hartog et al. 2004), Kuiper et al. (2006) also showed for these and for a fourth AXP (1E 2259+586) pulsed hard X-ray emission using archival RXTE data. That means that presently already for 4 of the 7 established AXPs hard X-ray emission has been detected and this can now be considered to be a common characteristic, which is not yet understood.

In this paper we focus on the AXP 4 U 0142+61. This AXP was discovered by the Uhuru X-ray observatory in the early seventies (Giacconi et al. 1972; Forman et al. 1978). The spin period of $8.7 \mathrm{~s}$ was found by Israel et al. (1994). They realised that the X-ray luminosity is too high to be explained by rotational energy loss. Like for the other AXPs, there is no proof for a companion, nor for an active (i.e. accreting) disk that could explain the high X-ray luminosity of $4 \mathrm{U} 0142+61$. The passive (i.e. non accreting) debris disk discovered by Wang et al. (2006) does not power the X-ray emission. The X-ray luminosities recently measured with e.g. XMM-Newton and Chandra are of the order of $10^{35} \mathrm{erg} \mathrm{cm}^{-2} \mathrm{~s}^{-1}(2-10 \mathrm{keV}$, see Patel et al. 2003; Göhler et al. 2005), assuming a distance of $3.6 \mathrm{kpc}$ (Durant and van Kerkwijk 2006a). The X-ray spectra $(0.5-10 \mathrm{keV})$ of AXPs are soft and are commonly fitted with a black-body and a power-law model. The inclusion of the power-law component is required to fit excess photons with energies above $\sim 3 \mathrm{keV}$. For $4 \mathrm{U} 0142+61$, the best fit parameters are a black-body temperature of $k T \sim 0.4 \mathrm{keV}$ and a power-law photon index of $\Gamma \sim 3.4$.

$4 \mathrm{U} 0142+61$ was detected by den Hartog et al. (2006) in hard X-rays up to $150 \mathrm{keV}$ in $1.6 \mathrm{Ms}$ of INTEGRAL observations (see also Kuiper et al. 2006). The $20-150 \mathrm{keV}$ flux was measured to be $(9.7 \pm 0.9) \times 10^{-11} \mathrm{erg} \mathrm{cm}^{-2} \mathrm{~s}^{-1}$. The total spectrum could be fitted with a power-law model with photon index $\Gamma=0.73 \pm 0.17$. They also revisited the Compton Gamma-Ray Observatory (COMPTEL) archives (0.75-30 MeV, see Schönfelder et al. 2002) and determined flux upper limits at the location of the AXP. These limits put constraints on the extrapolation of the hard X-ray power law. Assuming that the hard X-ray flux is stable, a spectral break has to occur in the hard X-ray regime below $\sim 750 \mathrm{keV}$ in order for the spectrum not to be in conflict with the COMPTEL upper limits. den Hartog et al. (2006) and Kuiper et al. (2006) were not able to measure such a break, but a hint was found with a $2.3 \sigma$ fit improvement when a high-energy cutoff was added to the power-law model by den Hartog et al. (2006). The indication for the cutoff was found at an energy of $73 \pm 15 \mathrm{keV}$.

A close connection may exist between the production of non-thermal hard X-rays and radio emission. However, until recently all AXPs were radio quiet. For AXP 1E 2259+58 the detection of radio emission has now been claimed by Malofeev et al. (2005), but the observations and analysis were difficult and confirmation is required. Halpern et al. (2005) discovered transient radio emission from the transient AXP XTE J1810-197 which appeared sharply modulated at the rotation period with peak flux densities $>1 \mathrm{Jy}$ (Camilo et al. 2006), orders of magnitude brighter than the reported upper limits for this or any other AXP. Gaensler et al. (2001) have observed 4U 0142+61 with the VLA $(1.4 \mathrm{GHz})$, but only a $5 \sigma$ upper limit of $0.27 \mathrm{mJy}$ could be extracted from the data.

4U 0142+61 was the first AXP for which an optical counterpart was discovered (Hulleman et al. 2000). Kern and Martin (2002) found that the optical emission is pulsed for a considerable fraction. Hulleman et al. (2000) showed for the first time that it is not possible to understand the optical and NIR measured fluxes with respect to the X-ray fluxes. There is an optical and NIR excess that can not be explained by the Rayleigh-Jeans tail from the X-ray black body. Moreover the optical and NIR emissions seem to be non thermal and exhibit more variability than seen in the X-rays (Hulleman et al. 2004; Israel et al. 2004; Morii et al. 2005; Durant and van Kerkwijk 2006c).

We present the first quasi-simultaneous multi-wavelength observation campaign to study $4 \mathrm{U} 0142+61$ from the radio up to hard X-rays.

\section{Multi-wavelength campaign}

For June-July 2005, 1 Ms INTEGRAL (20-300 keV) dedicated $4 \mathrm{U} 0142+61$ observations were scheduled. With these observations, we tried to get, nearly simultaneously, as much wavelength-band coverage of $4 \mathrm{U} 0142+61$ as possible. An approved 12 hour radio observation with the WSRT $(21 \mathrm{~cm})$ was rescheduled to overlap with the INTEGRAL observations. A regular RXTE (2-250 keV) monitoring observation also fell in the INTEGRAL time line. For an X-ray imaging observation a Target of Opportunity (ToO) was granted with Swift $(0.2-10 \mathrm{keV})$. Finally, optical and NIR observations were requested and approved in the Directors' Discretionary Time (DDT) on Gemini North. During two nights $4 \mathrm{U} 0142+61$ was imaged in the $K_{s}$ and $r^{\prime}$ bands. Unfortunately it was not possible to schedule the $K_{s}$ and the INTEGRAL observations contemporaneous (see Table 1).

\subsection{Hard X-rays: INTEGRAL}

The INTErnational Gamma-Ray Astrophysics Laboratory (INTEGRAL; Winkler et al. 2003) is ESA's currently operational hard X-ray/soft gamma-ray space telescope. For the study of AXPs the low-energy detector of IBIS, called ISGRI (20-300 keV, Lebrun et al. 2003), has proven itself to be of great importance. The serendipitous discovery of AXPs in the INTEGRAL energy band was a result of the combination of long exposure times and the $29^{\circ}$ wide FOV of IBIS. 
Table 1 Multi-wavelength campaign measurements

\begin{tabular}{lll}
\hline Obs & Time span & Exposure \\
\hline WSRT & July 2, 2005 & $12 \mathrm{~h}$ \\
Gemini $K_{s}$ & July 26, 2005 & $1125 \mathrm{~s}$ \\
Gemini $r^{\prime}$ & July 13, 2005 & $2400 \mathrm{~s}$ \\
Swift & July 11-12, 2005 & $7400 \mathrm{~s}$ \\
INTEGRAL & June 29-July 17, 2005 & $868 \mathrm{ks}$ \\
\hline
\end{tabular}

For this work we have analysed 1 Ms of observations of $4 \mathrm{U} 0142+61$ performed between June 29 and July 17, 2005. The data were screened for solar flares and erratic count rates resulting from passes through the Earth's radiation belts. After screening the net exposure was $868 \mathrm{ks}$ (Table 1). The observations consist of 265 pointings (Science Windows, ScWs) which can last up to one hour. The ScWs have been analysed separately with the official INTEGRAL software OSA 5.1 (see Goldwurm et al. 2003, for IBISISGRI scientific data analysis) in 20 energy bands between $20 \mathrm{keV}$ and $300 \mathrm{keV}$ with exponential binning. These analyses result in sky images for every $\mathrm{ScW}$ in 20 energy bands. The spectrum was built up by averaging the count rates from each $\mathrm{ScW}$, weighted by the variance. For the conversion into flux values, the spectrum was normalized to the known total Crab spectrum (nebula and pulsar) using a curved power law as determined by Kuiper et al. (2006).

$4 \mathrm{U} 0142+61$ is detected up to $230 \mathrm{keV}$ with a $3.0 \sigma$ significance in the $150-230 \mathrm{keV}$ energy band. The total spectrum shown in Fig. 1 was fitted with a power-law model resulting in a photon index $\Gamma=0.79 \pm 0.10$. The quality of the fit is good with a reduced chi square $\chi_{r}^{2}=1.12$ for 16 degrees of freedom. The $20-230 \mathrm{keV}$ flux is $(17.0 \pm 1.4) \times$ $10^{-11} \mathrm{erg} \mathrm{cm}^{-2} \mathrm{~s}^{-1}$.

Our new total spectrum shows that this AXP is now detected at even higher energies than reported earlier, without an indication for a spectral break. The hint for a break found by den Hartog et al. (2006) is not confirmed in this data set.

\subsection{Soft X-rays}

\subsubsection{Swift}

The Swift-XRT (0.2-10 keV; Burrows et al. 2005) observation was performed on July 11-12, lasting $8500 \mathrm{~s}$. Of this observation $7400 \mathrm{~s}$ of data were taken in the PhotonCounting mode and were analysed with the FTOOLS xrtpipeline, version build-14 under HEADAS 6.0 (Hill et al. 2005). Photons were extracted from an annular region ( 3 pixels inner radius, 30 pixels outer radius) in order to avoid pile-up contamination. Background spectra were taken from close-by source-free regions.

As mentioned in Sect. 1, AXP spectra in the X-ray domain $(<10 \mathrm{keV})$ can usually be fitted satisfactorily with a black-body and a power-law model. When we use this canonical model the fit results are: $N_{\mathrm{H}}=(1.01 \pm 0.10) \times$ $10^{22} \mathrm{~cm}^{-2} ; k T=(0.400 \pm 0.012) \mathrm{keV} ; \Gamma=2.7 \pm 0.3 ; \chi_{r}^{2}=$ 0.97 (dof $=573)$. These parameters yield an unabsorbed flux of $(3.9 \pm 0.2) \times 10^{-10} \mathrm{erg} \mathrm{cm}^{-2} \mathrm{~s}^{-1}$ in the $0.7-6.0 \mathrm{keV}$ band, or $(7.8 \pm 0.5) \times 10^{-11} \mathrm{erg} \mathrm{cm}^{-2} \mathrm{~s}^{-1}$ in the more commonly used 2-10 keV band. Such a model fits the measured X-ray spectrum well, but systematically overestimates the flux at the lower X-ray energies. Furthermore it seems meaningless for extrapolation to the NIR window. In particular, the soft power-law component dominates the blackbody component for energies less than $\sim 1.5 \mathrm{keV}$, meaning that also the $N_{\mathrm{H}}$ estimate is affected and estimated too high.

Alternatively, we used a double black-body model to fit the measured spectrum, yielding an acceptable fit $\left(\chi_{r}^{2}=\right.$ 0.98; dof $=573$ ) with an excellent fit at the lower X-ray energies, but underestimating the higher $\mathrm{X}$-ray energies $(>4.5 \mathrm{keV})$. The two temperatures are $0.38 \pm 0.02 \mathrm{keV}$ and $0.78 \pm 0.10 \mathrm{keV}$ and the $N_{\mathrm{H}}$ is $(0.61 \pm 0.03) \times$ $10^{22} \mathrm{~cm}^{-2}$. The unabsorbed flux is $(2.11 \pm 0.06) \times 10^{-10}$ erg cm ${ }^{-2} \mathrm{~s}^{-1}$ in the $0.7-6.0 \mathrm{keV}$ band and $(6.94 \pm 0.29) \times$ $10^{-11} \mathrm{erg} \mathrm{cm}^{-2} \mathrm{~s}^{-1}$ in the $2-10 \mathrm{keV}$ band. Arguably, this model is again canonical, but it is more appropriate for extrapolation to the NIR, which is shown in Fig. 1.

\subsubsection{Rossi-XTE}

$4 \mathrm{U} 0142+61$ monitoring data of the PCA (2-60 keV; Jahoda et al. 1996) on board the Rossi X-ray Timing Explorer (RXTE, Bradt et al. 1993) were used to create a phase-coherent timing solution valid during the multiwavelength campaign (see Gavriil and Kaspi 2002, for a detailed description and use of the AXP-monitoring campaign). This ephemeris is essential for INTEGRAL timing analysis (cf., den Hartog et al. 2007). In this work it is used for the WSRT observation in order to reduce the number of trials in the search for a (possible) weak pulsed radio signal. Data from RXTE-observation IDs 90076 and 91070 were analysed with the pulsar-timing software package TEMPO. ${ }^{1}$ The resulting ephemeris is valid between MJD 53251 and MJD 53619, with the following characteristics: Epoch MJD 53420.0, $v=0.1150929855(7) \mathrm{Hz}, \dot{v}=$ $-2.639(8) \times 10^{-14} \mathrm{~Hz} \mathrm{~s}^{-1}$ and $\ddot{v}=3(2) \times 10^{-23} \mathrm{~Hz} \mathrm{~s}^{-2}$.

\subsection{Optical and NIR: Gemini}

The field of $4 \mathrm{U} 0142+61$ was imaged in the $K_{s}$ band with the Near Infrared Imager (NIRI; Hodapp et al. 2003) on Gemini North, Hawaii, in the night of July 26th, 2005. NIRI has standard broad-band and narrow-band filters covering $1-5 \mu \mathrm{m}$.

\footnotetext{
${ }^{1}$ http://pulsar.princeton.edu/tempo.
} 
Fig. 1 Source-energy

distribution of $4 \mathrm{U} 0142+61$

from radio up to gamma-ray

energies. For observation

details, see Sect. 3. The WSRT

radio limits are shown for the

continuum emission (top arrow)

and the pulsed emission (lower

arrow). Extrapolations to lower

energies are shown for the

Swift-XRT double black-body

fit (dotted line) and for the

INTEGRAL power-law fit (dashed line). It can be seen that neither the soft X-ray nor the hard X-ray spectral fits extrapolate to the Optical and NIR fluxes. An optical-NIR excess orders of magnitude above these extrapolations is evident. The COMPTEL upper limits at $\mathrm{MeV}$ energies do not belong to the multi-wavelength campaign

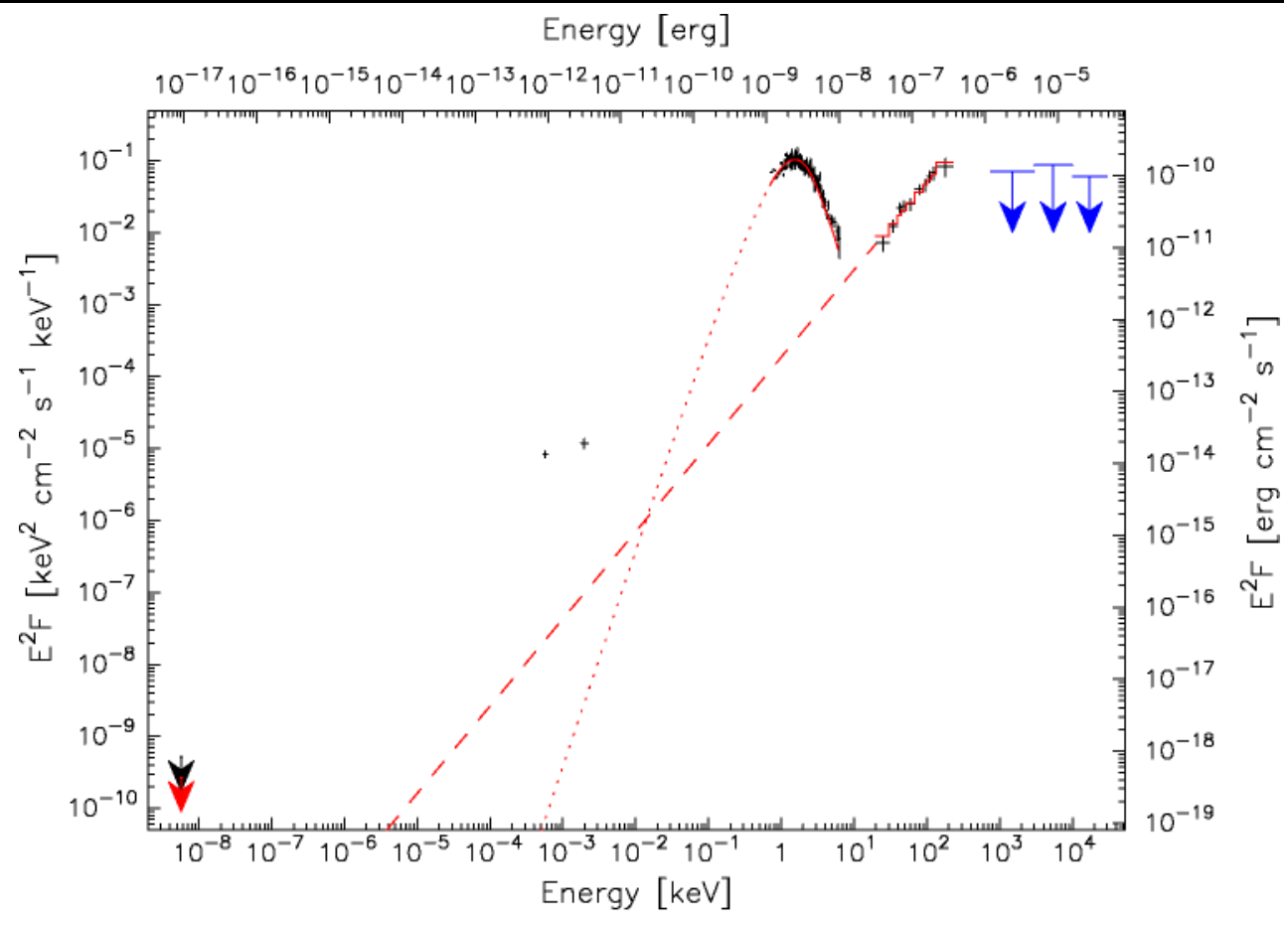

The final image was created by subtracting dark frames from each science frame, dividing by a flat field derived from the images themselves, and then aligning and stacking all the images. The photometry was measured using the PSF-fitting package DAOPhot (Stetson 1987), and calibrated against the photometry provided for several field stars in Hulleman et al. (2004). The $K_{s}$ magnitude was found to be $19.96(10)$.

Optical $r^{\prime}$-band images were obtained on the night of July 13th, 2005 using the Gemini-North Multi-Object Spectrograph (GMOS-N; Hook et al. 2004). We subtracted the bias and divided by screen flats, before stacking and photometering the images. For the calibration, the photometry listed in Hulleman et al. (2004) was used, interpolating between the $\mathrm{R}$ and V-bands using the relationship in (Smith et al. 2002). We find for $4 \mathrm{U} 0142+61 r^{\prime}=25.42(6)$, where the statistical uncertainty in the measurement and the uncertainty in the calibration of the photometry zero-point are similar.

\subsection{Radio: WSRT}

Using the Westerbork Synthesis Radio Telescope (WSRT) we have searched for both pulsed and unpulsed radio emission. An observation of 12 hour duration was carried out at a frequency of $1380 \mathrm{MHz}(\sim 21 \mathrm{~cm})$ with a bandwidth of $80 \mathrm{MHz}$. Using the synthesis data a map was made using standard routines in the MIRIAD ${ }^{2}$ package. In the resulting map (rms $\sim 30 \mu \mathrm{Jy}$ ) no source was detected at the location

\footnotetext{
${ }^{2}$ http://www.atnf.csiro.au/computing/software/miriad/.
}

of $4 \mathrm{U} 0142+61$ leading to a $3 \sigma$ upper limit on its flux of $\sim 90 \mu \mathrm{Jy}$. Simultaneously we also summed the signals from all 14 dishes of the WSRT coherently to form a so-called tied array. The data were then sent to the Pulsar Machine PuMa (Voûte et al. 2002) which formed a digital filter bank with 512 channels and a sampling time of $409.6 \mu \mathrm{s}$. As the dispersion measure in the direction of the source was unknown, we tried many trial dispersion measures and then folded each one with the RXTE ephemeris (see Sect. 2.2.2). Each resultant profile was then inspected to determine if there was a significant detection. We also performed a standard pulsar search analysis on the full data set. Neither the folding nor the search revealed any significant detection of radio pulsations. A $5 \sigma$ upper limit was determined at $77 \times \sqrt{(} d / 1-d) \mu \mathrm{Jy}$ where $d$ is the duty cycle of the pulsar. Using $d=0.5$, a typical value in the X-ray regime, the $3 \sigma$ upper limit is $\sim 46 \mu \mathrm{Jy}$. It has to be noted that for this analysis the whole observation was used. A finer analysis like performed by Halpern et al. (2005) and Camilo et al. (2006) who discovered the transient AXP XTE J1810-197 as a bright transient radio source in smaller intervals is still ongoing. However $4 \mathrm{U} 0142+61$ has not exhibited the same sort of transient behaviour in X-rays like XTE J1810-197, specially it has not shown any large outburst, and therefore the radio characteristics of these sources might be very different.

\section{Multi-wavelength source-energy distribution}

In Fig. 1 the quasi-simultaneous multi-wavelength SourceEnergy Distribution (SED) is presented covering roughly 10 
orders of magnitude in photon energy. In the lower left corner the WSRT $(1380 \mathrm{MHz})$ continuum (upper arrow) and timing (lower arrow) $2 \sigma$ upper limits are shown. The Gemini $K_{s}(2.15 \mu \mathrm{m})$ and $r^{\prime}(0.6 \mu \mathrm{m})$ data points are dereddened assuming $A_{V}=3.6$ as determined by Durant and van Kerkwijk (2006b). The Swift-XRT spectrum between $0.7 \mathrm{keV}$ and $6 \mathrm{keV}$ is corrected for absorption with $N_{\mathrm{H}}=$ $0.61 \times 10^{22} \mathrm{~cm}^{-2}$. Also shown is the corresponding double black-body fit (solid line), extrapolated towards lower energies (dotted line). The INTEGRAL flux values between $20 \mathrm{keV}$ and $230 \mathrm{keV}$ show the hard X-ray spectrum for this AXP. The power-law fit (solid line) is also extrapolated to lower energies (dashed line).

Significant flux variability has been reported in the optical, NIR and soft X-ray bands (Durant and van Kerkwijk 2006c), therefore in this quasi-simultaneous spectrum we can investigate better how the fluxes in the different bands relate to each other.

\section{Discussion}

The results for the different wave-band measurements render separately no surprises. The Swift-XRT spectrum shows a typical soft AXP spectrum and NIR and optical magnitudes are around the earlier reported values. The INTEGRAL spectrum is in agreement with previously found results, however, thanks to the high effective exposure in this dedicated observation the maximum energy up to which $4 \mathrm{U} 0142+61$ could be detected is now higher, namely $230 \mathrm{keV}$. The spectrum, described with a power-law model with photon index $\Gamma=0.79 \pm 0.10$ and luminosity $8.7 \times$ $10^{34} \mathrm{erg} \mathrm{s}^{-1}(20-100 \mathrm{keV}, d=3.6 \mathrm{kpc})$ can be compared with the spectral results reported earlier by den Hartog et al. (2006) using an independent data set: $\Gamma=0.73 \pm 0.17$ and luminosity $8.5 \times 10^{34} \mathrm{erg} \mathrm{s}^{-1}(20-100 \mathrm{keV}, d=3.6 \mathrm{kpc})$. Kuiper et al. (2006) derived $\Gamma=1.05 \pm 0.11$ and luminosity $8.1 \times 10^{34} \mathrm{erg} \mathrm{s}^{-1}(20-100 \mathrm{keV}, d=3.6 \mathrm{kpc})$. All these findings are within errors in agreement. Therefore there is no evidence for long-term time variability at hard X-ray energies yet.

den Hartog et al. (2006) published $2 \sigma$ flux upper limits for $4 \mathrm{U} 0142+61$ in the $0.75-30 \mathrm{MeV}$ window analysing COMPTEL data collected over the years 1991-2000. These observations are obviously not contemporaneous to the multi-wavelengths campaign reported here, but the apparent stability at hard X-ray energies seems to justify a direct comparison of the COMPTEL upper limits with the hard $\mathrm{X}$-ray spectrum measured with INTEGRAL. Thus assuming that the hard X-ray emission is stable, Fig. 1 clearly shows that the hard X-ray/soft gamma-ray spectrum of $4 \mathrm{U}$ $0142+61$ has to break between $\sim 200 \mathrm{keV}$ and $750 \mathrm{keV}$ in order not to be in conflict with the COMPTEL upper limits. If we were to assume that by chance $4 \mathrm{U} 0142+61$ was in a low state at $\mathrm{MeV}$ energies during the long COMPTEL observations, it would be remarkable that also for the other AXPs detected similarly by INTEGRAL (1E 1841-045 and 1RXS J1708-4009) no signal was found at MeV energies during the many observations that they were in the COMPTEL field-of-view spread over 10 years (Kuiper et al. 2006). We conclude that a drastic break is required.

The Swift-XRT and INTEGRAL spectra can not be fitted simultaneously with a two-component model consisting of i.e. a black-body plus only one power-law component. The excess of high-energy photons (w.r.t a single black-body spectrum) in the Swift spectrum can not be accounted for by the upcoming hard X-ray spectrum. This is evident in Fig. 1. The extrapolation of the hard X-ray spectral fit towards lower energies is already five times lower at $6 \mathrm{keV}$ than measured with Swift.

The relation of the optical and NIR flux values to the soft and hard X-ray spectra is still an enigma. It was noted earlier (e.g., Hulleman et al. 2004) that extrapolations of fits to the soft X-ray spectra to the optical and NIR bands are not consistent with the measured variable flux values. This is more than evident in Fig. 1, where we show the extrapolation of the double black-body fit, which reaches optical and NIR fluxes $\sim 4-5$ orders of magnitude lower than the measured values. The very hard X-ray spectra above $10 \mathrm{keV}$, originally interpreted as being of non-thermal origin, led to suggestions of a common origin as the likely non-thermal optical and NIR emissions. However, Fig. 1 unambiguously shows that also extrapolation of the power-law spectral shape measured at hard X-ray energies passes 2-3 orders of magnitude underneath the optical and NIR data points. The hard X-ray, soft X-ray, optical and NIR components seem to have different origins.

The discovery of the hard X-ray spectra of AXPs, stimulated new attempts to search for non-thermal radio emission possibly originating from the same sites and/or production mechanisms (e.g. synchrotron radiation) in the magnetar's magnetosphere. The WSRT upper limits at $1380 \mathrm{MHz}$ are however not constraining in this respect. The INTEGRAL power-law fit extrapolates also orders of magnitudes below the radio upper limits.

The physical interpretation of the hard X-ray spectrum measured by INTEGRAL is not evident. Assuming a distance of $3.6 \mathrm{kpc}$ as recently determined by Durant and van Kerkwijk (2006a), the 20-230 keV flux (Sect. 2.1) translates to a luminosity of $2.6 \times 10^{35} \mathrm{erg} \mathrm{s}^{-1}$, which exceeds the maximum luminosity available from rotational energy $\operatorname{loss}^{3}$ with a factor of $\sim 2000$. Moreover, the hard X-ray luminosity is comparable with the high soft $\mathrm{X}$-ray luminosity

\footnotetext{
${ }^{3} L_{\mathrm{RE}}=1.3 \times 10^{32} \mathrm{erg} \mathrm{s}^{-1}$ assuming a neutron star with $R=10 \mathrm{~km}$, $M=1.4 M_{\odot}$
} 
$\left(1.1 \times 10^{35} \mathrm{erg} \mathrm{s}^{-1}, 2-10 \mathrm{keV}\right)$ as measured with Swift (see Sect. 2.2.1).

Over the last two years theoretical attempts have been made trying to explain the new hard X-ray emission above $10 \mathrm{keV}$ from AXPs. A particularly interesting interpretation of the high-energy emission is the application of a magnetarcorona model by Thompson and Beloborodov (2005) and Beloborodov and Thompson (2007). In the last papers, a theoretical model is developed explaining the formation of a hot corona above the surface of a magnetar, so far the only model attempting to explain the multi-wavelength emission. In essence, the twisted magnetosphere acts as an accelerator that converts the toroidal-field energy to particle kinetic energy. They show numerically that the corona self organises quickly into a quasi-steady state, with a voltage of $\sim 1 \mathrm{GeV}$ along the magnetic field lines. Pair production occurs at a rate just enough to feed the electric current. Interestingly, the heating rate of the corona is $\sim 10^{36}-10^{37} \mathrm{erg} \mathrm{s}^{-1}$, in agreement with the observed persistent, high-energy output of magnetars. Furthermore, they deduce that the static twist will decay on a timescale of $1-10 \mathrm{yr}$, setting the scale for time variability to look for in the high-energy emission. The transition layer between the atmosphere and the corona is the likely source of the observed $\sim 100 \mathrm{keV}$ (e.g. bremsstrahlung) emission from magnetars. Finally, it is worth mentioning that the corona emits curvature radiation which can also supply the observed IR-optical luminosity. Of particular concern in the hot coronae model is the reported timescale of 1-10 yr. For 4U 0142+61 we do not see evidence for strong variability in the hard X-ray emission over the first years of INTEGRAL observations, nor over the longer period of RXTE monitoring observations. Future work will concentrate on this aspect, as well on detailed timing studies, including phase resolved spectroscopy. Obviously, as much INTEGRAL data as possible will be collected on 4U $0142+61$ and the other hard X-ray emitting AXPs to search for the break energy in the total spectrum, a key parameter in the discrimination between the different proposed models. More detailed analysis of this campaign and additional INTEGRAL observations will be presented in forthcoming papers by Rea et al. (2007) and den Hartog et al. (2007), respectively.

Acknowledgements We acknowledge the Director of Gemini for his DDT and also the Swift team for the ToO. The results in this paper are based on observations with INTEGRAL.

We acknowledge J. Vink for the use of private INTEGRAL Cas A data.

\section{References}

Beloborodov, A.M., Thompson, C.: Astrophys. J. (2007, accepted) Beloborodov, A.M., Thompson, C.: Astrophys. Space Sci. 308. DOI 10.1007/s10509-007-9318-x (2007)
Bradt, H.V., Rothschild, R.E., Swank, J.H.: Astron. Astrophys. Suppl. Ser. 97, 355 (1993)

Burrows, D.N., Hill, J.E., Nousek, J.A., et al.: Space Sci. Rev. 120, 165 (2005)

Camilo, F., Ransom, S., Halpern, J.P., et al.: Nature 442, 892 (2006)

den Hartog, P.R., Kuiper, L., Hermsen W., et al.: Astron. Telegr. 293, 1 (2004)

den Hartog, P.R., Hermsen, W., Kuiper, L., et al.: Astron. Astrophys. 451, 587 (2006)

den Hartog, P.R., Hermsen, W., Kuiper, L., et al.: Astron. Astrophys. (2007, in preparation)

Duncan, R.C., Thompson, C.: Astrophys. J. Lett. 392, L9 (1992)

Durant, M., van Kerkwijk, M.H.: Astrophys. J. 650, 1070 (2006a) (astro-ph/0606027)

Durant, M., van Kerkwijk, M.H.: Astrophys. J. 650, 1082 (2006b) (astro-ph/0606604)

Durant, M., van Kerkwijk, M.H.: Astrophys. J. 652, 576 (2006c)

Forman, W., Jones, C., Cominsky, L., et al.: Astrophys. J. Suppl. Ser. 38, 357 (1978)

Gaensler, B.M., Slane, P.O., Gotthelf, E.V., et al.: Astrophys. J. 559, 963 (2001)

Gavriil, F., Kaspi, V.M.: Astrophys. J. 567, 1067 (2002)

Giacconi, R., Murray, S., Gursky, H., et al.: Astrophys. J. 178, 281 (1972)

Göhler, E., Wilms, J., Staubert, R.: Astron. Astrophys. 433, 1079 (2005)

Goldwurm, A., David, P., Foshini, L., et al.: Astron. Astrophys. 411, L223 (2003)

Halpern, J.P., Gotthelf, E.V., Becker, R.H., et al.: Astrophys. J. Lett. 632, L29 (2005)

Hill, J.E., Angelini, L., Morris, D.C., et al.: Int. Soc. Opt. Eng. 5898, 325 (2005)

Hodapp, K.W., Jensen, J.B., Irwin, E.M., et al.: Publ. Astron. Soc. Pac. 115, $1388(2003)$

Hook, I.M., Jørgenson, I., Allington-Smith, J.R., et al.: Publ. Astron. Soc. Pac. 116, 425 (2004)

Hulleman, F., van Kerkwijk, M.H., Kulkarni, S.R.: Nature 408, 689 (2000)

Hulleman, F., van Kerkwijk, M.H., Kulkarni, S.R.: Astron. Astrophys. 416, 1037 (2004)

Israel, G.L., Mereghetti, S., Stella, L.: Astrophys. J. Lett. 433, L25 (1994)

Israel, G.L., Stella, L., Covino, S., et al.: IAU Symp. 218, 247 (2004)

Jahoda, K., Swank, J.H., Giles, A.B., et al.: Int. Soc. Opt. Eng. 2808, 59 (1996)

Kaspi, V.M.: Astrophys. Space Sci. (2007) this volume

Kern, B., Martin, C.: Nature 417, 527 (2002)

Kuiper, L., Hermsen, W., Mendez, M.: Astrophys. J. 613, 1173 (2004)

Kuiper, L., Hermsen, W., den Hartog, P.R., et al.: Astrophys. J. 645, 556 (2006)

Lebrun, F., Leray, J.P., Lavocat, P., et al.: Astron. Astrophys. 411, L141 (2003)

Malofeev, V.M., Malov, O.I., Teplykh, D.A., et al.: Astron. Rep. 49, $242(2005)$

Molkov, S., Cherepashchuck, A.M., Lutovinov, A.A., et al.: Astron. Lett. 30, 534 (2004)

Morii, M., Kawai, N., Kataoka, J., et al.: Adv. Space Res. 35, 1177 (2005)

Patel, S.K., Kouveliotou, C., Woods, P.M., et al.: Astrophys. J. 587, 367 (2003)

Rea, N., Turolla, R., Zane, S., et al.: (2007, submitted)

Revnivtsev, M.G., Sunyaev, R.A., Varshalovich, D.A., et al.: Astron. Lett. 30, 382 (2004)

Schönfelder, V., Aarts, H., Bennett, K., et al.: Astrophys. J. Suppl. Ser. 86, 657 (1993)

Smith, J.A., Tucker, D.L., Kent, S., et al.: Astron. J. 123, 2121 (2002) 
Stetson, P.B.: Publ. Astron. Soc. Pac. 99, 191 (1987)

Thompson, C., Duncan, R.C.: Mon. Not. Roy. Astron. Soc. 275, 255 (1995)

Thompson, C., Duncan, R.C.: Astrophys. J. 473, 322 (1996)

Thompson, C., Beloborodov, A.M.: Astrophys. J. 634, 565 (2005)

Voûte, J.L.L., Kouwenhoven, M.L.A., van Haren, P.C., et al.: Astron. Astrophys. 385, 733 (2002)

Wang, Z., Chakrabarty, D., Kaplan, D.L.: Nature 440, 772 (2006)
Winkler, C., Courvoisier, T.J.-L., Di Cocco, G., et al.: Astron. Astrophys. 411, L1 (2003)

Woods, P.M., Thompson, C.: In: Lewin, W., van der Klis, M. (eds.) Compact Stellar X-ray Sources. Cambridge Astrophysics Series, vol. 39, p. 547. Cambridge University Press, Cambridge (2006) (astro-ph/0406133) 